Cahiers $d u$ MONDE RUSSE

\section{Cahiers du monde russe}

Russie - Empire russe - Union soviétique et États indépendants

$51 / 1 \mid 2010$

Pierre le Grand et ses images de Rome

\title{
Les études antiquaires dans la bibliothèque de Pierre le Grand
}

Antiquarian studies from Peter the Great's library

\section{Daniela Gallo}

\section{(2) OpenEdition}

\section{Journals}

Édition électronique

URL : https://journals.openedition.org/monderusse/9173

DOI : 10.4000/monderusse. 9173

ISSN : $1777-5388$

Éditeur

Éditions de l'EHESS

Édition imprimée

Date de publication : 15 avril 2010

Pagination : 71-85

ISBN : 978-2-7132-2306-8

ISSN : $1252-6576$

Référence électronique

Daniela Gallo, «Les études antiquaires dans la bibliothèque de Pierre le Grand », Cahiers du monde russe [En ligne], 51/1 | 2010, mis en ligne le 10 mai 2013, consulté le 03 septembre 2022. URL : http:// journals.openedition.org/monderusse/9173; DOI : https://doi.org/10.4000/monderusse.9173

Ce document a été généré automatiquement le 3 septembre 2022

Tous droits réservés 


\title{
Les études antiquaires dans la bibliothèque de Pierre le Grand
}

\author{
Antiquarian studies from Peter the Great's library
}

\author{
Daniela Gallo
}

1 Ceux qui voudraient tirer des conclusions sur la passion antiquaire du tsar Pierre le Grand à partir de l'image gravée par Maria de Wilde [ill. 1] vers 1700 pour le catalogue des statuettes antiques de la collection de son père risqueraient, à notre avis, de se tromper ${ }^{1}$. Il est vrai que le souverain y est représenté lors de sa célèbre visite du 13 décembre 1697 au cabinet du marchand Jacob de Wilde, l'un des collectionneurs de pierres gravées et de monnaies parmi les plus réputés des dernières décennies du XVII siècle, que le tsar avait rencontré à l'Amirauté d'Amsterdam, où de Wilde était secrétaire et trésorier². Âgé de vingt-cinq ans, Pierre le Grand, une loupe à la main, est en train de regarder des pierres gravées ou des monnaies, et son hôte semble l'avoir momentanément distrait pour lui vanter les qualités de certains petits bronzes de sa collection. De nombreux livres remplissent les rayons des deux bibliothèques qui constituent le principal décor de cette pièce, tandis qu'un beau médaillier trône dans la grande niche du fond. Sur les côtés, deux globes occupent les étagères centrales, la partie supérieure des bibliothèques servant de présentoir pour la collection de statuettes en bronze - où l'on reconnaît aisément des copies en miniature du Laocoon et du Marc-Aurèle du Capitole - et des oushebtis égyptiens. Enfin, des instruments de mesure accrochés sur les deux panneaux extérieurs de ces meubles attirent encore notre regard, nous rappelant qu'en cette fin du XVII siècle, les collectionneurs d'antiquités ne dédaignaient pas de s'intéresser aux savoir-faire techniques. 


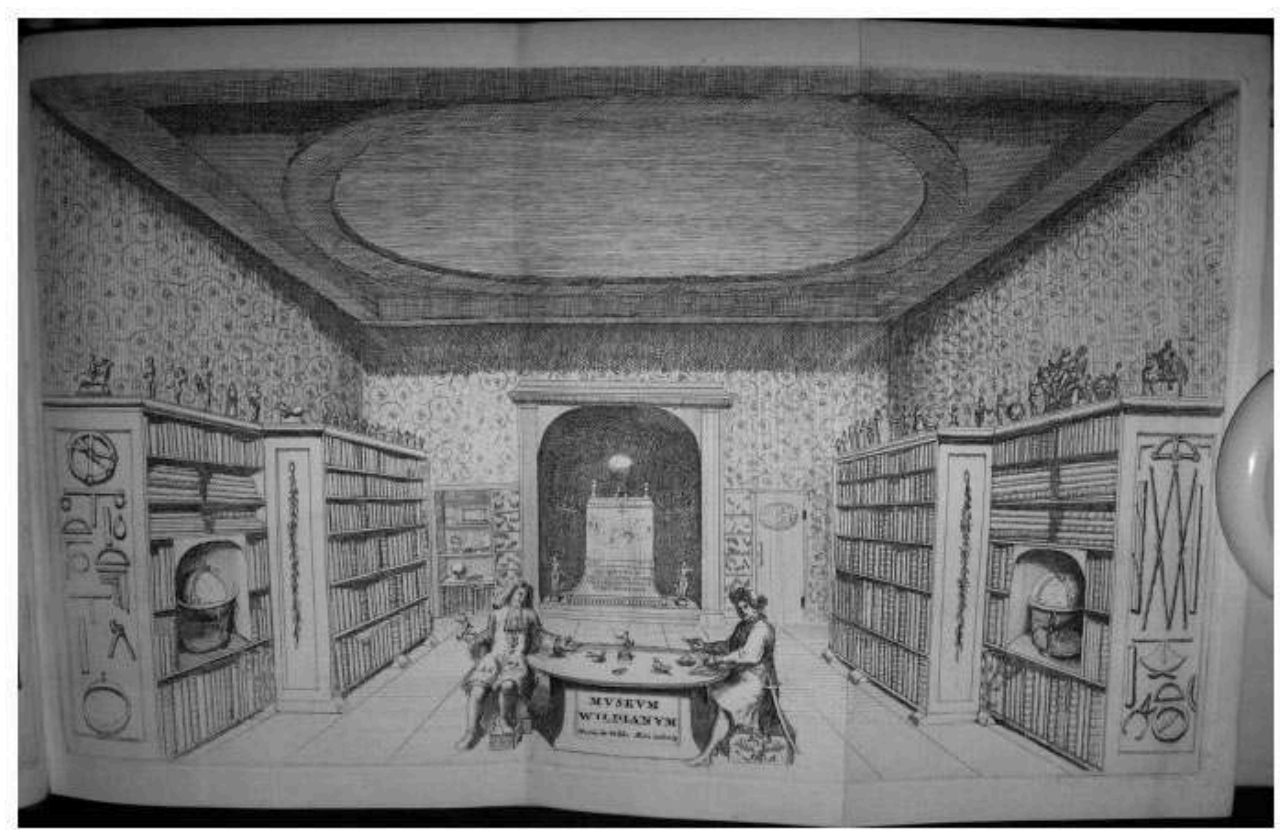

Maria de Wilde, Conversation piece entre Pierre le Grand et Jacob de Wilde, d'après Signa Antiqua e museo Jacobi de Wilde veterum poetarum carminibus illustrata et per Mariam filiam æri inscripta, Amsterdam : sumptibus autoris, 1700.

2 En effet, dans cette image-souvenir, sont au rendez-vous tous les ingrédients nécessaires à une présentation du tsar comme membre de la République des Lettres. Assis dans un cabinet-bibliothèque, le collectionneur et le souverain sont pris par une conversation savante qui, de l'antiquaria, pourrait rapidement passer à des questions d'ordre scientifique ou technique. Les deux curieux sont concentrés sur des objets, mais nous comprenons bien que les volumes qui les entourent peuvent tout autant fournir des réponses à leurs éventuelles interrogations que leur permettre de vérifier les hypothèses qu'ils viendraient à formuler. Il convient de se souvenir qu'en ce tournant du XVII e siècle, la nouvelle critique historique avait prôné l'utilisation du document, et surtout des objets, comme preuves irréfutables pour toute reconstitution historique, déclassant ainsi un savoir fondé exclusivement sur les connaissances livresques ${ }^{3}$. Selon ce témoignage de Maria de Wilde, lors de son premier voyage en Europe et grâce à sa rencontre avec Jacob de Wilde, le tsar était donc devenu un savant de la République des Lettres, passionné d'antiquaria et au courant des tendances de la nouvelle histoire.

3 Il en fut différemment vingt ans plus tard, si nous nous en tenons aux souvenirs de Frédérique Sophie Wilhelmine de Prusse, fille ainée du roi Frédéric-Guillaume et future margrave de Bayreuth, âgée de huit ans en 1717. Dans ses Mémoires, on lit que lorsque, au retour de son deuxième voyage en Europe, Pierre fit halte avec sa cour à Berlin, il lui fut montré

tout ce qu'il y avoit de remarquable [...], et entr'autres le cabinet de médailles et de statues antiques. Il y en avoit une parmi ces dernières, à ce qu'on m'a dit, qui représentoit une divinité païenne dans une posture fort indécente : on se servoit du temps des anciens Romains de ce simulacre pour parer les chambres nuptiales. On regardoit cette pièce comme très rare; elle passoit pour être l'une des plus belles qu'il y ait. Le Czar l'admira beaucoup et ordonna à la Czarine de la baiser. Elle voulut s'en défendre, il se fâcha et lui dit en allemand corrompu : Kop ab, ce qui signifie : je vous ferai décapiter si vous ne m'obéissez. La Czarine eut si peur qu'elle 
fit tout ce qu'il voulut. Il demanda sans façon cette statue et plusieurs autres au roi qui ne put les lui refuser. Il en fit de même d'un cabinet dont toute la boiserie étoit d'ambre. Ce cabinet étoit unique dans son espèce et avoit coûté des sommes immenses au roi Frédéric premier. Il eut le triste sort d'être conduit à Pétersbourg au regret de tout le monde. ${ }^{4}$

Des médailles et pierres gravées aux petits bronzes et aux priapées, le tsar Pierre avait continué à s'intéresser aux antiques mais, vingt ans après son premier voyage d'initiation à la culture européenne, c'étaient encore les curiosa qui retenaient son attention. Il fut sans doute influencé en cela par la réalité des cabinets qu'il avait visités. En Hollande, en Prusse, en Angleterre, en Écosse, au Danemark, mais en France aussi, dans ces premières décennies $\mathrm{du} \mathrm{XVIII}^{\mathrm{e}}$ siècle, les collections importantes de marbres antiques étaient toujours bien peu nombreuses ${ }^{5}$. Comme nous l'a rappelé récemment Olga Medvedkova $^{6}$, il fallut attendre les années 1718-1719 pour que l'architecte-pensionnaire Jurij Kologrivov envoyât d'Italie les premiers marbres antiques à Saint-Pétersbourg, les cadeaux berlinois n'étant rien d'autre que des petits bronzes ou des statuettes en terre cuite.

5 Aurait-il pu en être autrement ? Si nous considérons l'histoire des collections romaines d'antiques, nous ne pouvons que répondre par la négative. C'est, en effet, seulement en septembre 1724, quelques mois à peine avant la mort de Pierre le Grand, que les princes Odescalchi vendirent la prestigieuse collection de la reine Christine de Suède au roi et à la reine d'Espagne. Quant aux marbres Chigi, ils arrivèrent à Dresde en 1728, l'année où le roi de Pologne réussit à acheter trente sculptures au cardinal Alessandro Albani, accablé de dettes ${ }^{7}$. Or, en 1724, aucun agent russe, ou travaillant pour le tsar - tel que l'architecte romain Nicola Michetti, qui avait succédé à Le Blond comme " architecte général » du chantier de la nouvelle ville de Saint-Pétersbourg -, ne pouvait l'emporter face à la couronne espagnole, vu les liens privilégiés que la famille du pape Innocent XI Odescalchi entretenait avec cette dynastie. Le duc d'Orléans s'était mis sur les rangs dès 1713, mais avait échoué en 1721, après huit ans de négociations, alors que le couple royal espagnol emporta la mise en huit mois ${ }^{8}$. En outre, après son séjour à Saint-Pétersbourg, Michetti était rentré à Rome l'été 1723 et avait rapidement renoué avec le cercle du cardinal Ottoboni, pour lequel il travaillait déjà avant de se rendre en Russie, ce qui l'amenait à fréquenter le parti francophile de la ville pontificale ${ }^{9}$. Absorbé par ses grands projets urbanistiques et maritimes, le tsar ne s'était pas rendu en Italie : seule sa présence à Rome et le versement d'une somme d'argent plus importante que celle de 50000 écus avancée par Philippe V d'Espagne et par la reine Isabelle auraient éventuellement pu changer la donne.

6 Si Pierre le Grand ne fut pas un collectionneur averti de marbres antiques, fut-il en revanche un amateur de livres d'antiquaria? Les voyageurs britanniques qui, en ce début du XVIII ${ }^{\mathrm{e}}$ siècle, ne pouvaient pas acquérir des marbres antiques de qualité, revenaient de la péninsule avec des ersatz, des copies de bronze, grandeur nature ou en version réduite, des statues antiques les plus célèbres ou encore des dessins. Ce fut le cas pour George Parker, fils du lord chancelier Macclesfield, pour Thomas Coke, comte de Leicester, ou pour Richard Topham, riche célibataire, conservateur des archives de la Tour de Londres, qui avait été député de Windsor au Parlement dans les années 1698-1713 et qui se constitua une vraie encyclopédie de l'Antiquité en gravures et dessins ${ }^{10}$.

7 Tel ne semble pas avoir été le cas pour le tsar Pierre qui, à partir de 1703, fut surtout absorbé par la construction et l'embellissement de Saint-Pétersbourg. Ainsi, exception faite pour les guides de Rome, étudiés ici par Philippe Sénéchal ${ }^{11}$ - l’inventaire de la 
bibliothèque pétrovienne ne répertorie qu'une quinzaine de livres d'antiquaria. Bien évidemment, nous y trouvons les catalogues illustrés des collections de Jacob de Wilde, Signa antiqua (les statuettes), publié à compte d'auteur à Amsterdam en 1700, et Gemmce selectee, paru trois ans plus tard toujours à compte d'auteur ${ }^{12}$. Ces deux ouvrages entrèrent dans la bibliothèque du tsar en 1717 , lors de son second séjour à Amsterdam. Sur les feuilles de garde des Gemme selectoe, que Wilde avait dédiées au roi d'Espagne Charles III, Maria de Wilde a dessiné à la plume avec de l'encre noire et brune deux médaillons disposés l'un au-dessus de l'autre et entrelacés par un ruban, sur lequel on lit : « Jacob·de'Wilde·MDCCXVII-Maria'de·Wilde » [ill. 2]. À l'intérieur du premier médaillon, le Christ est en train de couronner le tsar; la légende clame: «CZAR.VIVE-BENEDICTUS.MAGNE ». Dans le second, Maria a dessiné une allégorie de la Fecunditas avec, dans l'exergue, la légende « FECUNDITATE-MAGNAE-CZARINAE-AUGUSTAE ». S'agit-il simplement de l'hommage rendu à l'empereur Pierre le Grand par ses anciens amis amstellodamois ou bien ce tribut était-il une façon détournée de proposer au tsar des iconographies pour une nouvelle médaille? La première hypothèse semblerait la plus plausible car dans le corpus des monnaies et médailles frappées sous le règne de Pierre nous n'avons pas trouvé trace de cette médaille. Quoi qu'il en soit, avec ces deux livres - auxquels il faudrait associer le catalogue sommaire de la collection numismatique, paru en 1692 et actuellement introuvable ${ }^{13}$-, Pierre le Grand emportait en Russie un cabinet de curiosités en papier, les portraits gravés de ses deux amis antiquaires et son propre portrait en membre de la République des Lettres. En effet, la conversation piece qui nous a intéressée au début se trouve dans le volume Signa antiqua, qui publie aussi le portrait de Maria de Wilde. 


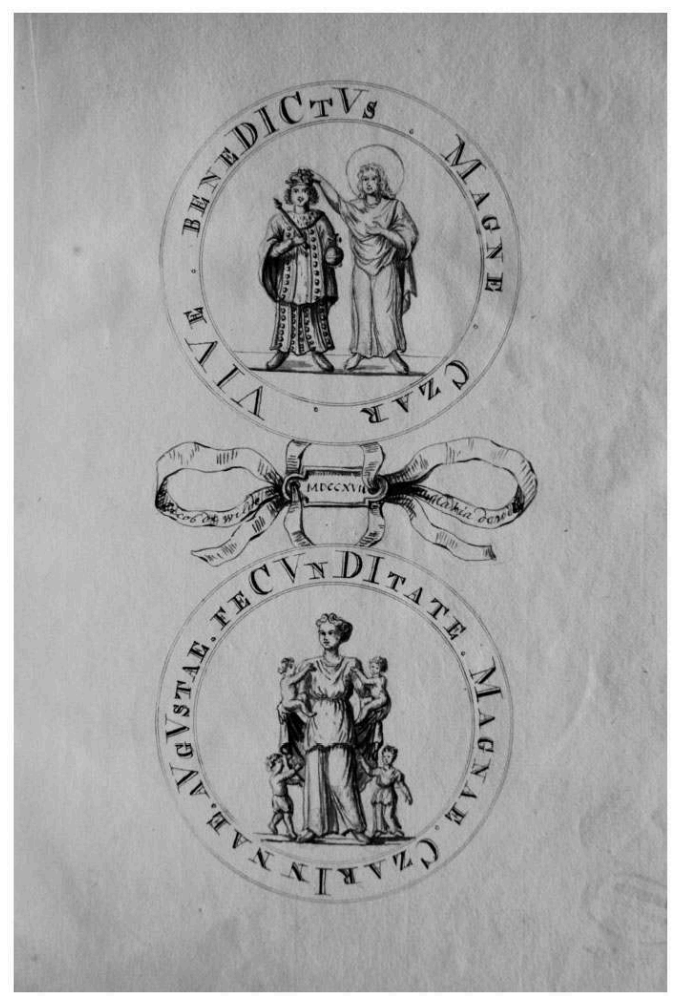

Maria de Wilde, Projet de médaille pour Pierre le Grand, 1717, dessin à la plume dans Gemmæ selectæ antiquæ e Museo Jacobi de Wilde, sive L. Tabulæ Diis Deabusque Gentilium ornatæ, per possessorem Conjecturis, Veterumque Poetarum carminibus illustratae, Amsterdam : sumptibus auctoris, 1703, feuilles de garde de l'exemplaire de la bibliothèque de Pierre le Grand.

Deux volumes sur les lampes des Anciens relèvent de ce même registre d'une antiquaria de cabinet. Il s'agit de la troisième édition, parue à Udine en 1653, du très volumineux et très érudit ouvrage du philosophe péripatéticien, médecin et naturaliste d'origine génoise Fortunio Liceti ${ }^{14}$, et d'un recueil, non daté, de trente planches, en grande partie gravées par Pietro Sante Bartoli ${ }^{15}$. Grâce à l'ex-libris, nous savons qu'en 1659, l'exemplaire du De Lucernis de Liceti, un vrai classique des études antiquaires du XVII siècle, appartenait au protonotaire apostolique de la cathédrale d'Anvers, le patricien bruxellois Guillaume van Hamme ; ce qui laisserait à penser que ce volume fut acheté dans les Flandres ou en Hollande. Aux dires de certains, le tsar maîtrisait le latin et, quoi qu'il en soit, il pouvait disposer de traducteurs. Cet ouvrage ne pouvait pas ne pas l'intéresser. Avant de prendre en compte les formes et l'iconographie des centaines d'objets sur lesquels est fondée son analyse, l'auteur répertorie en effet les matériaux de combustion utilisés dans l'Antiquité et tente d'expliquer pourquoi, lorsqu'on ouvrait les tombeaux antiques, les fouilleurs voyaient briller une flamme, comme si, depuis des siècles, ces lampes n'avaient jamais cessé leur fonction.

Le recueil de Bartoli, un mélange en partie issu de la réutilisation de planches de Le Antiche lucerne sepolcrali figurate de Bellori paru en $1691^{16}$, est d'un ordre tout à fait différent. Dans ce volume, les dix-sept lampes, pour la plupart en bronze, partagent la scène avec des camées et des intailles. On y trouve aussi un petit buste de Jupiter en bronze, un buste en marbre d'Antinoüs, deux statuettes en bronze de Vénus et d'Hercule et une monnaie de Flavie-Hélène. Plutôt qu'à un ouvrage érudit, nous avons à faire à un répertoire de modèles, formels et iconographiques, dont la page de titre est conçue 
comme une plaque gravée et encadrée. Sans doute réalisé dans les premières décennies du XVIII ${ }^{e}$ siècle, ce volume témoigne d'une période de transition, dans laquelle l'éditeur qui est probablement Francesco Bartoli, le fils et héritier de Pietro Sante - essaye de répondre à la demande des collectionneurs de curiosité, qui continuaient à raffoler d'objets bizarres et de petites dimensions, mais en renouvelant la formule de sa présentation grâce à une syntaxe plus classicisante.

Répertorié comme livre d'histoire naturelle, alors que les catalogues de Maria de Wilde avaient été classés comme livres d'antiquaria ${ }^{17}$, le Museum Wormianum du médecin danois Ole Worm ne pouvait pas manquer dans la bibliothèque du fondateur de la Kunstkamera de Saint-Pétersbourg, lequel fut aussi le premier collectionneur d'objets archéologiques en Russie ${ }^{18}$. Cet ouvrage dense et savant avait été publié à titre posthume en 1655 par le fils de Worm, Willum, médecin lui aussi. L'impression commença à Leyde chez les Elzévirs durant l'association de Jean et Daniel, ce dernier se réservant la moitié de l'édition lorsqu'il s'associa avec son parent Louis Elzévir. De la sorte, il en existe des exemplaires, dont celui du tsar Pierre, qui affichent Amsterdam comme lieu d'édition ${ }^{19}$. Ce volume est l'un des témoignages les plus accomplis de la culture des Wunderkammern qui avait eu tant de succès dans l'Europe du nord dès la seconde moitié du XVI e siècle, voire constitue - selon Alain Schnapp - «le premier traité général des matériaux archéologiques et ethnologiques $»^{20}$. En effet, exception faite pour les monnaies, les objets, antiques et modernes, y sont répertoriés selon leur matériau et non selon leur genre ou leur technique. En outre, les antiques du Museum Wormianum qui, en 1654, fut légué par le collectionneur au roi du Danemark, ne relevaient pas uniquement de l'Antiquité classique. Ils comprenaient aussi bien des instruments provenant du Groenland que des objets russes, tels une croix en argent, des monnaies, des manuscrits et des chaussures ${ }^{21}$.

11 Quant à Antiquarum Statuarum Urbis Romce de Giovan Battista Cavalieri, un graveur trentin du cercle des princes-évêques Madruzzo, il fut le plus important - et le premier répertoire illustré des statues antiques des collections romaines publié au $\mathrm{XVI}^{\mathrm{e}} \mathrm{siècle}^{22}$. L'exemplaire pétersbourgeois issu de la bibliothèque de Pierre le Grand, où il avait été classé comme livre d'iconographie, correspond à la seconde édition, composée de deux livres et dédiée au cardinal Ludovico Madruzzo, qui parut en 1585. Bien que de mauvaise qualité, ces cent planches in- $4^{\circ}$, dont les dessins auraient été réalisés par Cavalieri dans les années 1555-1574, offrent un bon panorama des plus importantes collections romaines de marbres antiques de cette époque, c'est-à-dire des collections vaticanes et capitolines, Cesi, Farnèse, Este, Capranica et Della Valle. L'ensemble est organisé selon un critère topographique qui, des jardins du Belvédère, au Vatican, conduit le lecteur aux Trophées de Marius, sur l'Esquilin, en lui faisant faire un long détour par la villa fuori porta du pape Jules III et en le guidant ensuite d'une colline à l'autre de la Ville éternelle. Mais, comme nous l'avons rappelé, un bon siècle après sa parution, à Saint-Pétersbourg l'ambitieux propos de Cavalieri avait été oublié et, de guide illustré des collections romaines d'antiques à la Renaissance, ces deux livres des statues de G.B.Cavalieri n'étaient désormais rien de plus qu'une source iconographique.

Les ouvrages de Giovan Pietro Bellori et de Pietro Sante Bartoli, imprimés à Rome dans les années 1690, qui figurent dans la bibliothèque de Pierre le Grand avaient été d'une ambition bien différente. Veteres Arcus Augustorum Triumphis Insignes ex Reliquiis quae Romce adhuc supersunt [ill. 3 et 4] - présent en deux exemplaires et classé parmi les livres d'iconographie ${ }^{23}-$, Admiranda Romanarum Antiquitatum ac Veteris Sculpturee Vestigia 
Anaglyphico Opere elaborata ex Marmoreis exemplaribus quae Romee adhuc exstant ${ }^{24}$ [ill. 5] et Gli Antichi Sepolcri, overo mausolei Romani et Etruschi, trovati in Roma \& in altri luoghi celebri ${ }^{25}$ constituaient des répertoires sur les arcs de triomphe de l'Urbs, sur les reliefs antiques que l'on pouvait voir aussi bien in situ sur ces mêmes arcs ou sur d'autres bâtiments en ruine que dans les collections privées ou au Capitole. Ils offraient un mélange de peintures, mosaïques, urnes, sarcophages, camées et inscriptions provenant de tombeaux fouillés à Rome et aux alentours à partir de la fin du xvI siècle. Il y avait aussi les recueils sur les reliefs des colonnes Trajane ${ }^{26}$ et Antonine ${ }^{27}$, dont les premières éditions avaient été publiées respectivement en 1672 et 1676, mais nous n'avons pu les consulter ${ }^{28}$.

3

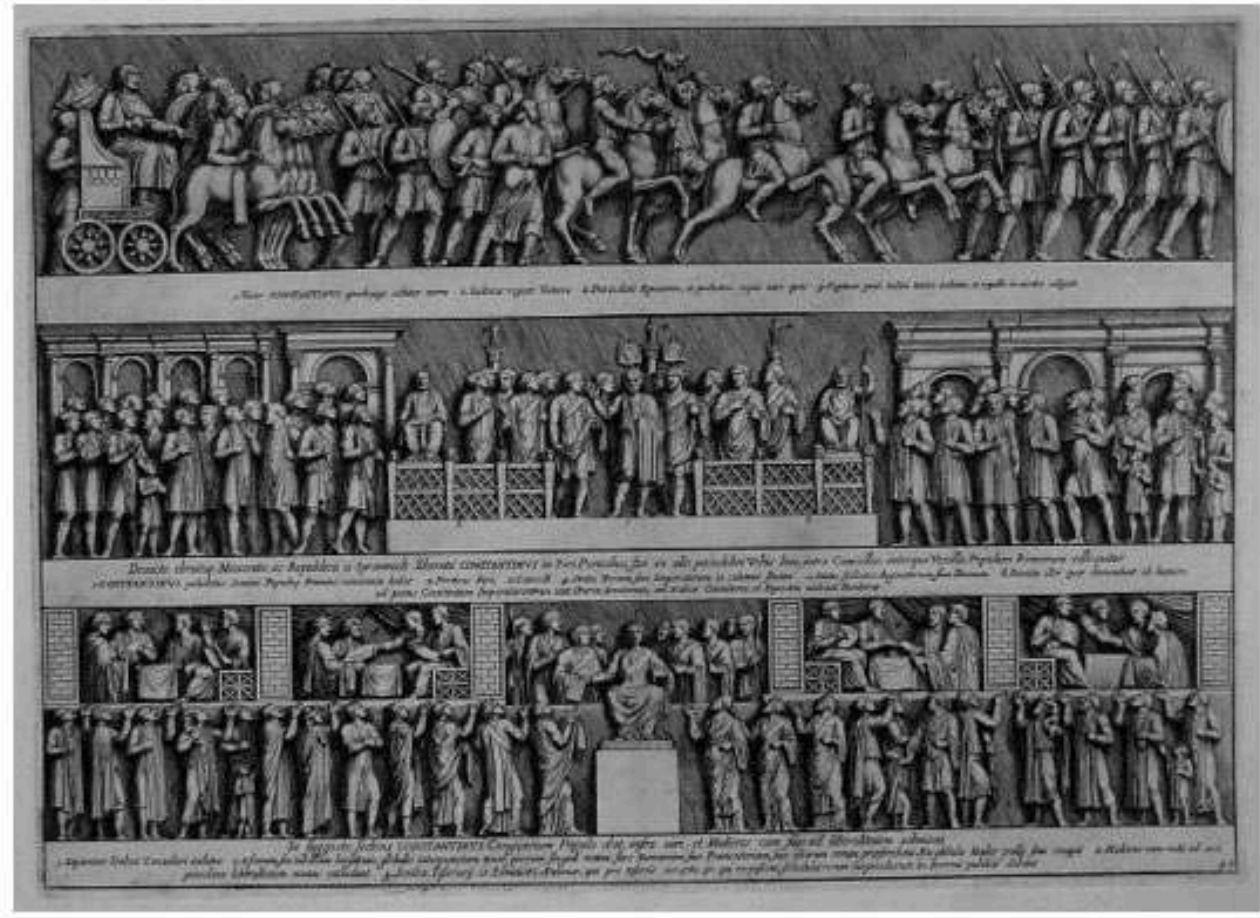

Giacomo De Rossi, Arc de Constantin, d'après Veteres Arcus Augustorum Triumphis Insignes ex Reliquiis quæ Romæ adhuc supersunt cum imaginibus triumphalibus restituti antiquis nummis notisque 1o. Petri Bellorii illustrati nunc primum per lo : lacobum De Rubeis æneis typis vulgati, Rome, ad Templum Sanctæ Mariæ de Pace, cum priviligio Sum. Pontificis, 1690. 


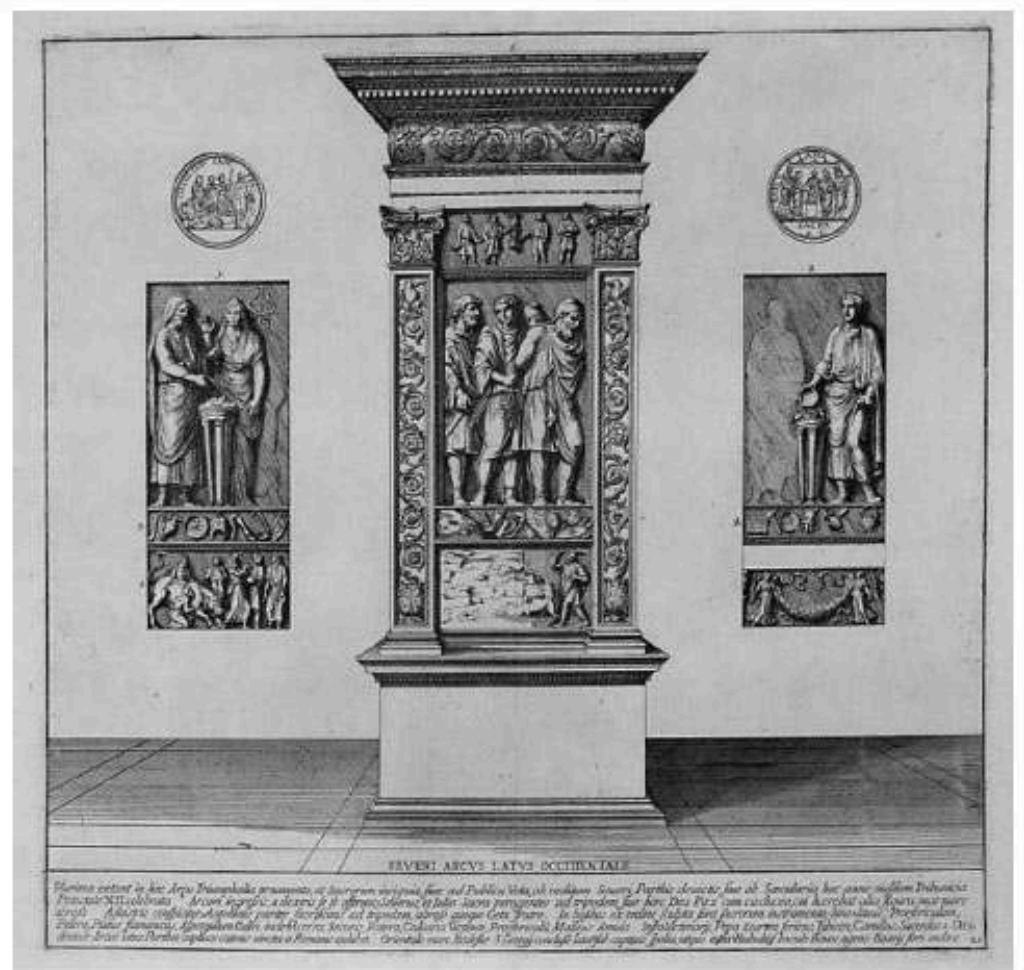

Giacomo De Rossi, Arc de Septime Sévère, d'après Veteres Arcus Augustorum Triumphis Insignes ex Reliquiis quæ Romæ adhuc supersunt cum imaginibus triumphalibus restituti antiquis nummis notisque lo. Petri Bellorii illustrati nunc primum per 10 : Iacobum De Rubeis æneis typis vulgati, Rome, ad Templum Sanctæ Mariæ de Pace, cum priviligio Sum. Pontificis, 1690.

5

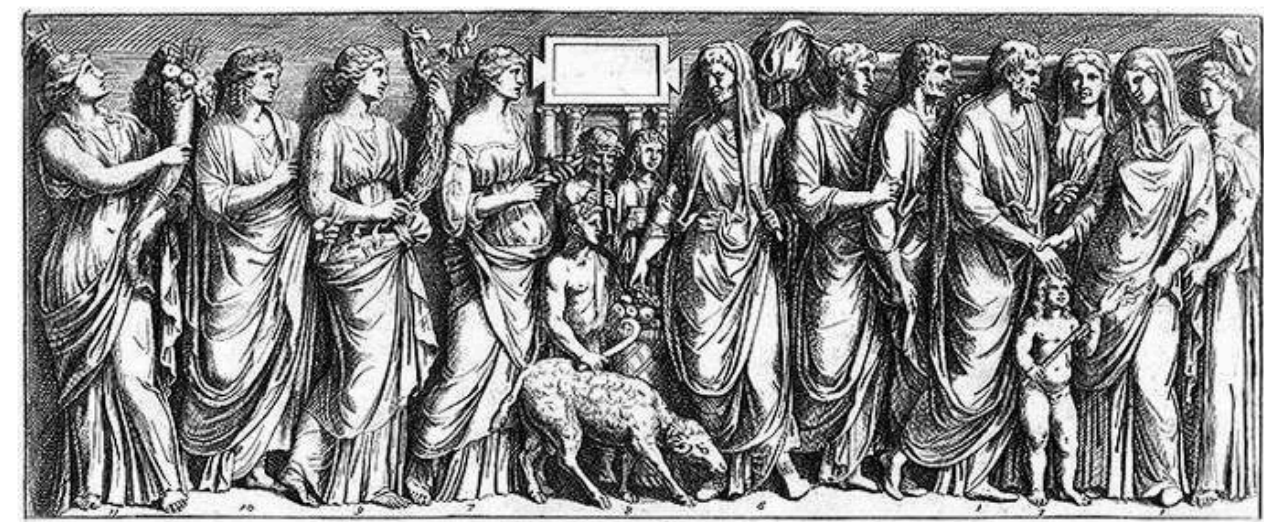

$$
\text { ispors }
$$

NVPTIAE

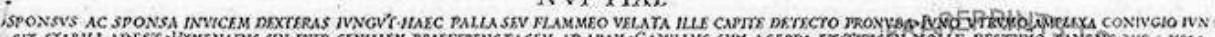

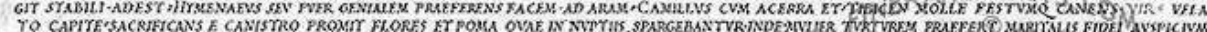

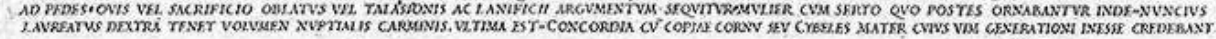

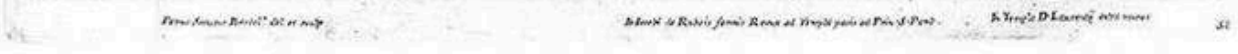

Pietro Sante Bartoli, Devant de sarcophage représentant une scène de mariage, d'après Admiranda Romanarum Antiquitatum ac Veteris Sculpturæ Vestigia Anaglyphico Opere elaborata ex Marmoreis exemplaribus quæ Romæ adhuc exstant, Rome : ad Templum S. a M. a e Pace, cum Privil. Summi Pont. et Licentia Superiorum, 1693. 
13 Nous le savons, tous ces ouvrages furent des best-sellers de l'édition romaine du $\mathrm{XVII}^{\mathrm{e}}$ siècle. Néanmoins, Olga Medvedvoka ${ }^{29}$ a prouvé que le volume sur la Trajane et les Admiranda furent achetés à Paris par l'agent officiel du tsar dans la capitale française, Konon Zotov, alors que l'on aurait plus facilement crédité de ces achats l'architecte romain Nicola Michetti. Zotov qui, pour justifier ces achats, écrivait à Pierre que les images des reliefs de la Trajane auraient été "très utile[s] aux peintres d'histoire ${ }^{30}$. Vincenzo Farinella a justement attiré l'attention sur le fait que ce fut grâce aux échafaudages que Louis XIV fit monter jusqu'au sommet de la Trajane pour mouler les reliefs destinés aux artistes de l'Académie de France à Rome que l'antiquaire et le graveur purent étudier de près la frise et corriger ainsi les observations d'Alfonso Chacón. De la sorte, Bellori et Bartoli dédièrent leur édition au roi de France, « il Traiano della Francia $»^{31}$. Le volume dut circuler rapidement et en de nombreux exemplaires audelà des Alpes, même si à Rome il ne fut mis en vente que chez l'imprimeur, Giovanni Giacomo De Rossi. En 1697, vingt-cinq ans après sa parution, il était devenu presque introuvable : le surintendant des bâtiments, Colbert de Villacerf, dut beaucoup insister auprès de La Teullière, alors directeur de l'Académie de France à Rome, pour en obtenir un exemplaire. Quoi qu'il en soit, le nom de Bellori était bien connu au nord des Alpes. Ses livres avaient fait l'objet de comptes rendus dans les périodiques savants dès 1673 et lorsque, après son décès en février 1696, sa collection d'antiques fut mise en vente, ce fut le prince électeur de Brandebourg qui se porta acquéreur ${ }^{32}$. Il est fort probable que Pierre le Grand ait entendu évoquer le nom de l'antiquaire romain lors de sa visite à l'«Antiquitaeten-Cammer» de Berlin, où se trouvaient alors les deux cents antiques environ qui avaient fait partie du cabinet Bellori avant que le roi Frédéric-Guillaume $\mathrm{I}^{\mathrm{er}}$ n'en cédât une partie à Auguste le Fort en échange de soldats pour son armée.

14 À Saint-Pétersbourg, les éditions de Bellori, Bartoli et De Rossi suscitèrent le plus grand intérêt. Preuve en est le projet de traduction en russe des Admiranda et des Veteres Arcus, étudié par Irina Hmelevskih dans ce numéro. Du reste, les édifices mêmes de la ville et leurs décors témoignent de l'influence que ces répertoires eurent sur les artistes, et non seulement à l'époque de Pierre le Grand. Zotov avait-il compris tout seul l'importance de ces ouvrages? Nous aimerions mieux connaître le milieu qui fut le sien à Paris. Certes, la qualité excellente des planches de Bartoli - qui figurent encore aujourd'hui parmi les meilleures estampes de reproduction d'après des sculptures - et de De Rossi pour l'architecture, la clarté du parti éditorial, thématique, et la maniabilité de ces volumes en firent des instruments de premier ordre dans une ville en construction, où la demande était énorme pour toute sorte d'artistes, sculpteurs, architectes, décorateurs ou peintres.

Enfin, dans une bibliothèque réalisée en grande partie pendant les deux premières décennies du XVIII ${ }^{\mathrm{e}}$ siècle ne pouvait pas manquer Raccolta di Statue Antiche e Moderne que l'éditeur romain Domenico De Rossi avait fait paraître en 1704 avec les explications du patricien toscan Paolo Alessandro Maffei ${ }^{33}$. Malheureusement, il ne nous a pas été donné de voir l'exemplaire en trois volumes de Pierre le Grand. Or, ce recueil de cent soixante et une planches au burin et à l'eau-forte reproduit les statues antiques les plus célèbres et les mieux conservées que l'on pouvait alors voir à Rome et Florence ainsi que les grands chefs-d'œuvre de Michel-Ange, du Bernin, de l'Algarde et du Fiammingo François Du Quesnoy. On y trouve aussi le Jonas que Lorenzetto avait réalisé d'après des dessins de Raphaël dans les années 1516-1520 pour la chapelle Chigi de Sainte-Marie-du-Peuple. Et, bien qu'encore fort exotiques aux yeux des artistes contemporains, y sont reproduites les rares statues égyptiennes des collections romaines et de l'archevêché de Tivoli. On s'en 
rend compte rapidement : en regardant ces planches, il ne fallait plus aller jusqu'à Rome ou à Florence pour se faire une idée de la grande statuaire antique gréco-romaine et de la sculpture italienne des XVI ${ }^{\mathrm{e}}$ et XVII siècles. Comme l'a dejà noté Sergej Androsov ${ }^{34}$, ce fut sans doute sur la base de ces reproductions que, en 1720, par l'entremise de Michetti, le tsar commanda à Rome des copies de l'Hercule Farnèse, du Gladiateur Borghèse, du Gaulois mourant et d'une Vénus. Il commanda aussi un modello en cire du Constantin du Bernin et s'enquit du prix de la fonte d'un cheval comme celui du Marc-Aurèle du Capitole, deux sculptures reproduites dans le recueil de Maffei.

Néanmoins, lorsque nous comparons ce fonds d'antiquaria avec ceux d'autres bibliothèques contemporaines, telles celle du duc de Brunswick et Wolfenbüttel dont, il y a une quinzaine d'années, Margaret Daly Davis a livré un catalogue critique ${ }^{35}$, l'exiguïté de la collection pétersbourgeoise saute immédiatement aux yeux. Exception faite de Selecta numismata antiqua du fonds De Wilde, nous n'y trouvons aucun ouvrage de numismatique, alors que les monnaies et médailles antiques avaient suscité un grand nombre de vocations aux XVI et $\mathrm{XVII}^{\mathrm{e}}$ siècles, et cela tant au nord qu'au sud de l'Europe. La même constatation s'impose pour les travaux sur les pierres gravées, complètement absents si l'on exclut Gemmae selectce du même cabinet amstellodamois. Dans la bibliothèque de Pierre le Grand, l'impasse sur l'épigraphie fut complète, et la cause n'est certes pas à chercher dans le latin. L'absence des grands ouvrages de Bosio ${ }^{36}$ et de Ciampini $^{37}$ sur les antiquités chrétiennes de Rome peut se justifier peut-être par des raisons religieuses et diplomatiques. En revanche, nous ne comprenons pas celle des grandes publications sur la peinture antique, tel le recueil de Bellori et Bartoli sur le sépulcre des Nasoni ${ }^{38}$ - présent seulement sous forme d'ersatz dans Beschryving de François Deseine, publiée en 1704, comme nous l'explique dans ce volume Philippe Sénéchal ${ }^{39}$ - ou le best-seller de Franciscus Junius, De pictura veterum libri tres, paru à titre posthume à Rotterdam en $1694^{40}$. Or, à la fin du XVII siècle, surtout à Rome, la peinture et les mosaïques antiques avaient tenu le devant de la scène tant du point de vue de la documentation des œuvres trouvées dans les fouilles que de leur conservation.

On dirait que les achats de livres d'antiquaria pour Pierre le Grand furent faits sans un projet précis, sinon éventuellement celui de fournir des modèles aux artistes ou de constituer un fond pour les collections de la Kunstkamera. En visitant les pays de l'Europe occidentale, le tsar avait bien senti que l'Antiquité gréco-romaine était le terreau commun de la culture européenne, tant du point de vue de l'histoire que des lettres et des arts. Ainsi, en voulant faire de sa nouvelle capitale une ville moderne et européenne, il lui fallait emprunter ce nouveau langage. Mais il n'est pas sûr que Pierre le Grand eut une vraie passion pour les antiques. Sa Kunstkamera est plutôt une chambre de naturalia et artificialia, comme si, nouvel empereur du Saint-Empire romain, il avait eu besoin de rassembler des pièces chargées d'une forte aura à transmettre à sa descendance ${ }^{41}$. La modernité en architecture et dans le décor ne pouvait se fonder, en revanche, que sur le vocabulaire et la syntaxe antiques, d'où les livres romains illustrés. Une fois traduites, certaines de ces sources auraient aussi pu proposer des modèles pour l'élaboration d'une nouvelle iconographie impériale. Mais il ne fut pas possible de concrétiser tous ces changements. Il fallut attendre encore une quarantaine d'années pour que, sous le règne de Catherine II, le modèle gréco-romain se répandît en force sur les berges de la Neva. Depuis, Pierre le Grand, fondateur de la nouvelle Russie, veille sur les destinées de sa ville, imperator triomphant sur son cheval cabré, sublime portrait à l'antique que Falconet livra à la Grande Catherine en 1782. 


\section{NOTES}

1. Voir Renée Kistemaker, Natalja Kopaneva, Annemiek Overbeek, éds., Peter de Grote en Holland: Culturele en wetenschappelijke betrekkingen tussen Rusland en Nederland ten tijde van tsaar Peter de Grote, catalogue de l'exposition (Amsterdam, Historisch Museum, 17 décembre 1996-13 avril 1997), Bussum: Thoth et Amsterdams Historisch Museum, 1996, p. 161 , cat. 37.

2. Voir Lodewijk-J. Wagenaar, « Jacob de Wilde, 1645-1721, an Amsterdam Collector», in Peter Berghaus, éd., Numismatische Literatur 1500-1864: Die Entwicklung der Methoden einer Wissenschaft, Wolfenbütteler Forschungen, 64, 1995, p.99-116; Julia Kagan, "Gesneden Stenen uit de Collectie van Nicolaas Chevalier en andere Nederlandse Verzamelaars », in Kistemaker, Kopaneva, Overbeek, éds., Peter de Grote en Holland, p. 67-81; Marianne Maaskant-Kleibrink, « Humanistische Gemmen in der Verzameling van Jacob de Wilde ", in ibid, p. 82-86; et Joaneath Spicer, "An "Antique" Brass Candlestick in the Shape of Hercules by Peter Vischer the Younger and Workshop ", The Journal of the Walters Art Museum, 63, 2005 (2008), p. 65-71.

3. Voir à ce propos Mario Rosa, «I depositi del sapere : biblioteche, accademie, archivi ", in Pietro Rossi, éd., La memoria del sapere: Forme di conservazione e strutture organizzative dall'antichità a oggi, Milan : Laterza, 1988, p. 165-209, et surtout p. 188-196 ; Francis Haskell, History and his Images: Art and the Interpretation of the Past, New Haven-Londres: Yale University Press, 1993, p.131-216; Peter N. Miller, éd., Momigliano and Antiquarianism: Foundations of the Modern Cultural Sciences, Toronto-Buffalo-Londres: University of Toronto Press, UCLA, 2007.

4. Mémoires de Frédérique Sophie Wilhelmine Margrave de Bayreuth, scur de Frédéric le Grand, depuis l'année 1706 jusqu'à 1742, écrits de sa main, préface de Pierre Gaxotte de l'Académie française et notes de Gérard Doscot, P.: Mercure de France, 1967, p. 62-63. Le cabinet d'ambre se trouve à Tsarskoje Selo. Il vient d'être restauré et complété.

5. Parmi ces exceptions, on retiendra la collection de Thomas, huitième comte de Pembroke qui, au début des années 1720 , réunit à Wilton House une série complète de bustes d'empereurs romains - dont certains étaient, toutefois, modernes - à partir de pièces achetées lors de la vente Mazarin à Paris et chez les princes Giustiniani à Rome. Cf. Jonathan Scott, The Pleasures of Antiquity: British Collectors of Greece and Rome, New HavenLondres : Yale University Press, 2003, p. 39-49.

6. Olga Medvedkova, «La bibliothèque d'architecture de Pierre le Grand : entre Curiosité et Passion", Cahiers du Monde russe, 47 (3), 2006, p. 467-502, 487. Voir aussi Sergej O. Androssov, "Sculptuur ", in Kistemaker, Kopaneva, Overbeek, éds., Peter de Grote en Holland, p. 247 ; idem, « Peter der Große als Sammler bildender Kunst », in Brigitte Buberl, Michael Dückershoff, éds., Palast der Wissens : Die Kunst- und Wunderkammer Zar Peters des Großen, Munich : Hirmer Verlag, 2003, II, p. 187-188.

7. Voir Daniela Gallo, «Euvrer pour la grandeur de Rome au XvIII siècle: Les collectionneurs d'antiques », in Benno Schubiger, Dorothea Schwinn Schürman, Cecilia Hurley, éds., Collections et pratiques de la collection en Suisse au XVIII siècle. Actes du colloque de Bâle, 16-18 octobre 2003, Genève : Slatkine, 2007, p. 489-511, 495. 
8. Voir, entre autres, Casimir Stryienski, La galerie du régent, Philippe, duc d'Orléans, P. : Goupil, Manzi et Joyant, 1913, p. 16-30 ; Ferdinand Boyer, « Les antiques de Christine de Suède à Rome ", Revue Archéologique, Ve série, XXXV, janvier-juin 1932, p. 254-267; Miguel Angel Elvira Barba, «El Palacio y la Colección de Esculturas », Cristina de Suecia en el Museo del Prado, catalogue de l'exposition (Madrid, musée du Prado, 30 octobre 1997-11 janvier 1998), Madrid: Ministerio de Educación y Cultura, 1997, p. 39-61; Pilar Silva Maroto, «La escultura clásica en las colecciones reales : de Felipe II a Felipe V », in El Coleccionismo de escultura clásica en España, actes du colloque (Madrid, musée du Prado, 21-22 mai 2001), Madrid : Museo nacional del Prado, 2001, p. 26-29.

9. Élève de Carlo Fontana, Nicola Michetti (1680 ou 1681-1758) avait été recommandé au tsar comme peintre de perspectives par Jurij Kologrivov sur les conseils du cardinal Pietro Ottoboni lui-même. Il partit pour la Russie début mai 1718. Voir John A. Pinto, "Nicola Michetti and Ephemeral Design in Eighteenth-Century Rome", in Henry A. Millon, éd., Studies in Italian Art and Architecture $15^{\text {th }}$ through $18^{\text {th }}$ Centuries, Memoirs of the American Academy in Rome, 35, 1980, p. 289-322; Idem, "Nicola Michetti and Eighteenth-Century Architecture in Saint Petersburg ", in Henry A. Millon, Susan Scott Munshower, éds., An Architectural Progress in the Renaissance and Baroque Sojourns In and Out of Italy: Essays in Architectural History Presented to Hellmut Hager on his Sixty-sixth Birthday, Papers in Art History from the Pennsylvania State University, VIII, II ${ }^{\mathrm{e}}$ partie, p. 527-565 ; Fiorella Pansecchi et Giorgio Falcidia, « Giovanni Sorbi, un pittore senese a Roma (e una nota su Nicola Michetti) ", in Arte Collezionismo Conservazione. Scritti in onore di Marco Chiarini, Florence et Milan: Giunti et Ente Cassa di Risparmio di Firenze, 2004, p. 370-371.

10. Voir Louisa Connor, « Richard Topham et les artistes du cercle Imperiali », in Joselita Raspi Serra, François de Polignac, éds., La Fascination de l'antique, 1700-1770: Rome découverte, Rome inventée, catalogue de l'exposition (Lyon, Musée de la civilisation galloromaine, 20 décembre 1998-14 mars 1999), Paris-Lyon: Somogy et Musée de la civilisation gallo-romaine, 1998, p. 52-54 ; Scott, The Pleasures of Antiquity, p. 57-61.

11. Philippe Sénéchal, « Les guides de la Rome antique dans la bibliothèque de Pierre le Grand », dans ce volume.

12. Signa Antiqua e museo Jacobi de Wilde veterum poetarum carminibus illustrata et per Mariam filiam ceri inscripta, Amsterdam : sumptibus autoris, 1700 ; Gemmce selectce antiquce e Museo Jacobi de Wilde, sive L. Tabulce Diis Deabusque Gentilium ornatee, per possessorem Conjecturis, Veterumque Poetarum carminibus illustratae, Amsterdam: sumptibus auctoris, 1703, in E.I. Bobrova, éd., Biblioteka Petra I: Ukazatel'-spravočnik [La Bibliothèque de Pierre $\mathrm{I}^{\mathrm{er}}$. Catalogue et index], L. : BAN, 1978, les deux volumes sont répertoriés aux $\mathrm{n}^{\circ} 1642$ et 1640.

13. Il s'agit de Selecta numismata antiqua ex museo Jacobi de Wilde, Amsterdam : sumptibus auctoris, 1692, répertorié sous le n 1641 dans Bobrova, éd., Biblioteka Petra I.

14. Fortunio Liceti, De Lucernis Antiquorum Reconditis Libb. Sex: in quibus earum recens inventarum adhuc ardentium observationes multce primum asseruntur: Aliorum opiniones omnes de ipsarum attributis dein expenduntur: earum causce, proprietates, differenticeque singuloe deinceps ex rei natura deteguntur: Pluresque dubitationes e traditis emergentes denuum eluuntur. Explicatis diligenter abditissimis qucestionibus de ignum causis, origine, varietate, duratione, motu, \& extinctu: De Antiperistati, Fumo, Cinere, Sentore, Mistis incombustilibus, Brutorum funeribus, \& alijs Naturce arcanis. Declaratisque plurimis Antiquitatis ritibus Lychnos supra centum effigiantibus: Cum indicibus locupletissimis, Udine: ex Typographia Nicolai 
Schirattii, 1653. Aumentibus Superioribus, prostant Patavij apud Franciscum Bolzettam. Selon le colophon, la date de parution serait en revanche 1652. Voir Bobrova, éd., Biblioteka Petra I, cat. 1268. Sur Liceti et son ouvrage, dont la première édition avait été publiée à Venise en 1621, voir Antoine Schnapper, Le Géant, la licorne, la tulipe : Collections et collectionneurs dans la France du XVII siècle, I, Histoire et histoire naturelle, P. : Flammarion, 1988, p. 169-171 ; Ingo Herklotz, Cassiano Dal Pozzo und die Archäologie des 17. Jahrhunderts, Munich: Hirmer Verlag, 1999, p. 158-162 ; Rosanna Bosso, « Lucerne », in Evelina Borea et Carlo Gasparri, éd., L'Idea del Bello: Viaggio per Roma nel Seicento con Giovan Pietro Bellori, catalogue de l'exposition (Rome, Palazzo delle Esposizioni et ex-Teatro dei Dioscuri, 29 mars-26 juin 2000), Rome : De Luca, II, p. 516-517; Philippe Malgouyres, « La petite sculpture en bronze, laboratoire du retour à l'antique ", à paraître in Daniela Gallo, Neville Rowley, éds., Imiter ce qui a disparu: Les artistes modernes face aux lacunes de l'héritage antique, actes du séminaire (Rome, Académie de France à Rome, 6-7 mars 2009).

15. Raccolta di Varia Antichità e Lucerne Antiche intagliate la maggior parte da Pietro Santi Bartoli, Rome, con licenza de' Superiori, in Bobrova, éd., Biblioteka Petra I, cat. 907.

16. Le Antiche lucerne sepolcrali figurate raccolte dalle cave sotterranee e grotte di Roma, nelle quali si contengono molte erudite memorie, disegnate, ed intagliate nelle loro forme da Pietro Santi Bartoli, divise in tre parti con le osservazioni di Gio: Pietro Bellori, Rome : Gio. Francesco Buagni, 1691. Réimprimé à Rome en 1704 et 1729, cet ouvrage avait été traduit en latin par Alexander Duker pour le dernier volume du Thesaurus Graecarum Antiquitatum de Jacobus Gronovius (Abraham van Goorle), paru à Leyde, chez VanDer Aa, en 1702. La même année, une autre édition latine par Lorenz Beger avait été publiée à Berlin. Il sera repris dans le volume XII de la nouvelle édition du Thesaurus Grcecarum Antiquitatum de Gronovius par Albert-Henri de Sallengre, publiée à Venise, chez B. Javarina et J.B. Pasquali, en 1737.

17. Dans les deux volumes sur les lampes, en revanche, nous n'avons trouvé aucune indication de classement.

18. Museum Wormianum. Seu Historia rerum rariorum, tam naturalium, quam artificialium, tam domesticarum, quam exoticarum, qua Hafnice Danorum in cedibus Auctoris servantur. Adornata ab OLAO WORM, Med. Doct. \&, in Regia Hafniensi Academia, olim Professore publico. Variis \& accuratis Iconibus illustrata, Amsterdam : apud Ludovicum \& Danielem Elzeviros, 1655, Bobrova, éd., Biblioteka Petra I, cat. 1653. Sur cet ouvrage, voir Alain Schnapp, La conquête du passé: Aux origines de l'archéologie, P. : Éditions Carré, 1993, p. 160-177. Sur la collection d'antiquités sibériennes de Pierre le Grand, voir, en revanche, Jelena F. Korolkowa, «Die Sibirische Sammlung Peters I.", in Buberl, Dückershoff, éds., Palast der Wissens, II, p. 201-209.

19. Voir Alphonse Willems, Les Elzevier: Histoire et annales typographiques, Bruxelles: G. A. Van Trigt, 1880, p. 192, cat. 772 [édition consultée, reprint Nieuwkoop : B. De Graaf, 1974].

20. Schnapp, La conquête du passé, p. 174. Sur la culture des Wunderkammern, voir, entre autres, Patricia Falguières, Les chambres des merveilles, P. : Bayard, 2003.

21. Museum Wormianum, respectivement p. 351, 360, 384 et 385 pour les objets russes et p. 366 pour les instrumenta groenlandais.

22. Antiquarum Statuarum Urbis Romce Primus et Secundus Liber Ludovico Madrucio S. R. E. Card. Amplissimo Dic. Io. Baptista De Cavalleriis Authore, Bobrova, éd., Biblioteka Petra I, cat. 1005. Sur cet ouvrage, voir Thomas Ashby, « Antiquæ statuæ urbis Romæ », Papers of 
the British School at Rome, 9, 1920, p.107-158; Liliana Menta, in Laura Dal Prà, éd., I Madruzzo e l'Europa, 1539-1658: I principi vescovi di Trento tra Papato e Impero, catalogue de l'exposition (Trente, Castello del Buonconsiglio et Riva del Garda, Chiesa dell'Inviolata, 10 juillet-31 octobre 1993), Milan-Florence : Charta, 1993, p. 328, cat. 70 ; Margaret Daly Davis, Archäologie der Antike, aus den Beständen der Herzog August Bibliothek, 1500-1700, catalogue de l'exposition (Wolfenbüttel, Zeughaus der Herzog August Bibliothek, 16 juillet-2 octobre 1994), Wiesbaden: Harrassowitz Verlag, 1994, p.122-124, cat.7.5; Paola Pizzamano, «Le traduzioni dall'antico », in Paola Pizzamano, éd., Giovanni Battista Cavalieri: Un incisore trentino nella Roma dei Papi del Cinquecento, Villa Lagarina 1525-Roma 1601, Rovereto : Nicolodi, 2001, p. 23-24.

23. Veteres Arcus Augustorum Triumphis Insignes ex Reliquiis quce Romce adhuc supersunt cum imaginibus triumphalibus restituti antiquis nummis notisque Io. Petri Bellorii illustrati nunc primum per Io: Iacobum De Rubeis ceneis typis vulgati, Rome, ad Templum Sanctce Marice de Pace, cum priviligio Sum. Pontificis, 1690. Dans Bobrova, éd., Biblioteka Petra I, cat. 1454, l'ouvrage est répertorié sous le nom de Giovanni Giacomo Rubens. Un autre exemplaire avec traductions en russe est conservé à la bibliothèque de l'Académie des Sciences sous la cote I. 6. 109.

24. Admiranda Romanarum Antiquitatum ac Veteris Sculpturce Vestigia Anaglyphico Opere elaborata ex Marmoreis exemplaribus quce Romce adhuc exstant in Capitolio cedibus hortisque virorum principum ad antiquam elegantiam a Pietro Sancti Bartolo delineata incisa in quibus plurima ac prceclarissima ad Romanam historiam ac veteres mores dignoscendos ob oculos ponuntur notis Io. Petri Bellorii illustrata Hoc omnium, quae extant nobilissima Romance magnitudinis monumenta ad perenne antiquitatis studium, ac decus cura, sumptibus, ac typis edita a Joanne Jacobo de Rubeis, restituit auxit Dominicus de Rubeis Chalcographus Anno MDCXCIII, Rome : ad Templum S.a M.a e Pace, cum Privil. Summi Pont. et Licentia Superiorum. Dans Bobrova, éd., Biblioteka Petra I, cat.1451, l'ouvrage est répertorié sous le nom de Giovanni Giacomo Rubens.

25. Gli Antichi Sepolcri, overo Mausolei Romani et Etruschi, trovati in Roma \& in altri luoghi celebri ; nelli quali si contengono molte erudite Memorie : raccolti, disegnati, \& intagliati da Pietro Santi Bartoli, Rome : nella stamperia di Antonio de Rossi dietro a San Silvestro in Capite in strada della Vite, 1697. Le volume porte le cachet de la bibliothèque de 1740. Voir Bobrova, éd., Biblioteka Petra I, cat. 905.

26. Colonna eretta dal Senato, e Popolo Romano all'Imperatore Traiano Augusto nel suo Foro in Roma. Scolpita con l'historie della guerra dacica la prima e la seconda espeditione, e vittoria contro il re Decebalo. Nuovamente disegnata, et intagliata da Pietro Santi Bartoli. Con l'espositione latina d'Alfonso Ciaccone, compendiata nella vulgare lingua sotto ciascuna immagine, accresciuta di medaglie, inscrittioni, e trofei, da Gio. Pietro Bellori. Con diligente cura, e spesa ridotta a perfettione, e data in luce da Gio. Giacomo de Rossi dalle sue stampe in Roma, alla Pace con Privilegio del S. Pontefice.

27. Colonna Antoniniana Marci Aurelii Antonini Augusti rebus gestis insignis Germanis simul et Sarmatis gemino bello devictis ex S.C. Romce in Antonini Foro ad viam Flaminiam erecta ac utriusque belli imaginibus anaglyphice insculpta nunc primum a Petro Sancti Bartolo iuxta delineationes in Bibliotheca Barberina adservatas a se cum antiquis ipsius columnce signis collatas, cere incisa et in lucem edita cum notis excerptis et declarationibus Io : Petri Bellorii.

28. Bobrova, éd., Biblioteka Petra I, cat. 1023 pour la Trajane (édition non datée) et 1024 pour l'Antonine (édition de 1704). 
29. Medvedkova, « La bibliothèque d'architecture de Pierre le Grand... », p. 496.

30. Lettre citée par Medvedkova, ibid.

31. Vincenzo Farinella, « Bellori e la Colonna Traiana », in Borea, Gasparri, éds., L'Idea del Bello, II, p. 589-602. Sur cet ouvrage, voir aussi Ingo Herklotz, «Bellori, Fabretti, and Trajan's Column ", in Janis Bell, Thomas Willette, éds., Art History in the Age of Bellori: Scholarship and Cultural Politics in Seventeenth-Century Rome, Cambridge: Cambrige University Press, 2002, p. 127-144 et 328-333.

32. C'est dans le Giornale dei Letterati di Roma de 1673 que furent publiés des comptes rendus du volume sur la Colonne Trajane (p. 13 s.) et de Fragmenta vestigii veteris Romce ex lapidibus Farnesianis, parus cette même année (p. 125 s.). Sur le cabinet de Bellori, voir Gerald Heres, «Die Sammlung Bellori: Antikenbesitz eines Archäologen im 17. Jahrhundert », Études et Travaux, X, 1978, p. 6-38 ; Idem, « Bellori collezionista: Il Museum Bellorianum », in Borea, Gasparri, éds., L'Idea del Bello, II, p. 499-501 ; Elena Vaiani, « Le antichità di Giovan Pietro Bellori : storia e fortuna di una collezione », Annali della Scuola Normale Superiore di Pisa, Classe di Lettere e Filosofia, 4e série, VII (1), 2002, p. 85-152.

33. Raccolta di Statue Antiche e Moderne date i luce sotto $i$ gloriosi auspici della Santità di N. S. Papa Clemente XI da Domenico De Rossi illustrata colle sposizioni a ciascheduna immagine di Paolo Alessandro Maffei Patrizio Volterrano e Cav. di S. Stefano e della Guardia pontificia, Rome : stamperia della Pace, 1704. Bobrova, éd., Biblioteka Petra I, cat. 1446. Sur cet ouvrage, voir Mouna Mékouar, Galerie d'art, lieu de savoir, mémoire de maîtrise sous la direction de Daniela Gallo et Alain Mérot, université Paris IV-Sorbonne, 2002-2003.

34. Androssov, « Peter der Große als Sammler bildender Kunst », in Buberl, Dückershoff, éds., Palast der Wissens, p. 188-189.

35. Daly Davis, Archäologie der Antike.

36. Antonio Bosio, Roma Sotterranea. Opera postuma compita, disposta e accresciuta dal M. R. P. Giovanni Severani da S. Severino, ...nella quale si tratta de' sacri cimiterii di Roma ... nuovamente visitati e riconosciuti dal Sign. Ottavio Pico, ...Publicata dal commendatore Fr. Carlo Aldobrandino, Rome: G. Facciotti, 1632. Réédité en italien à Rome, chez L. Grignani, en 1650, l'ouvrage de Bosio avait aussi paru en latin par les soins de Paolo Aringhi à Rome, expensis B. Diversini et Z. Masotti, dès l'année suivante. Cette édition latine fut encore réimprimée à Cologne, et mise en vente à Paris, chez. P. Léonard, en 1659.

37. Giovanni Giustino Ciampini, Vetera Monimenta, in quibus prcecipue Musiva Opera Sacrarum, Profanarumque Ædium Structura, ac nonnulli antiqui Ritus, Dissertationibus, Iconibusque illustrantur, Rome: ex typographia Joannis Jacobi Komarek Bohemi, apud S. Angelum Custodem, 1690-1699. 2 vol.

38. Le Pitture antiche del Sepolcro de' Nasoni nella via Flaminia disegnate ed intagliate alla similitudine degli Antichi Originali da Pietro Santi Bartoli, descritte \& illustrate da Gio: Pietro Bellori, Rome : per Gio. Battista Bussotti, 1680.

39. Sénéchal, « Les guides de la Rome antique dans la bibliothèque de Pierre le Grand », dans ce volume.

40. Franciscus Junius, De pictura veterum libri tres, tot in locis emendati, \& tam multis accessionibus aucti, ut plane novi possint videri: accedit catalogus, adhuc ineditus, architectorum, mechanicorum, sed prcecipue pictorum, statuariorum, ccelatorum, tornatorum, aliorumque artificum \& operum quce fecerunt, secundam seriem litterarum digestus, Rotterdam : typis Regneri Leers, 1694. 
41. Sur le contenu de la Kunstkamera, voir, entre autres, Oleg Neverov, «De Collecties van de Kunstkamera van Peter de Grote », in Kistemaker, Kopaneva, Overbeek, éds., Peter de Grote en Holland, p. 18-21; Debora J. Meijers, «De Kunstkamera van Peter de Grote: De Nederlandse bijdrage aan een nieuw type museum ", ibidem, p. 22-36; Buberl, Dückershoff, éds., Palast der Wissens, 2 vol. Mais, plus généralement, sur les enjeux des Wunderkammern fondamental est l'essai de Falguières, Les chambres des merveilles, P.: Bayard, 2003.

\section{RÉSUMÉS}

Résumé

En visitant les pays de l'Europe occidentale, Pierre le Grand avait bien senti que l'Antiquité grécoromaine était le terreau commun de la culture européenne, tant du point de vue de l'histoire que des lettres et des arts. Il emprunta ce nouveau langage aussi bien aux artistes venus d'Occident, qu'aux œuvres et livres acquis par ses agents. Moins étudiée que sa Kunstkammer, sa bibliothèque - et en particulier le choix des livres sur les antiques - nous a permis de mieux évaluer l'intérêt du tsar pour cette culture antique. Si nous y trouvons de grands classiques des études antiquaires des $\mathrm{XVI}^{\mathrm{e}}$ et $\mathrm{XVII}^{\mathrm{e}}$ siècles, nous n'en sommes pas moins frappés, au stade actuel de nos connaissances, par les lacunes.

Antiquarian studies from Peter the Great's library

Peter the Great's visits to Western European countries clearly showed him that Greco-Roman antiquity was the common basis of European culture in respect to both history and the arts. He borrowed this new language from artists coming from the West as well as works and books acquired by his agents. Less studied than his Kunstkammer, his library - and especially its collection of books about antiquities - has enabled us to give a better evaluation of the tsar's interest in that ancient culture. If we find major sixteenth- and seventeenth-century classics of antiquarian studies, we are also struck - at the present state of our knowledge - by the fact that works are missing.

\section{AUTEUR}

\section{DANIELA GALLO}

Université Pierre Mendès France-Grenoble 2 